
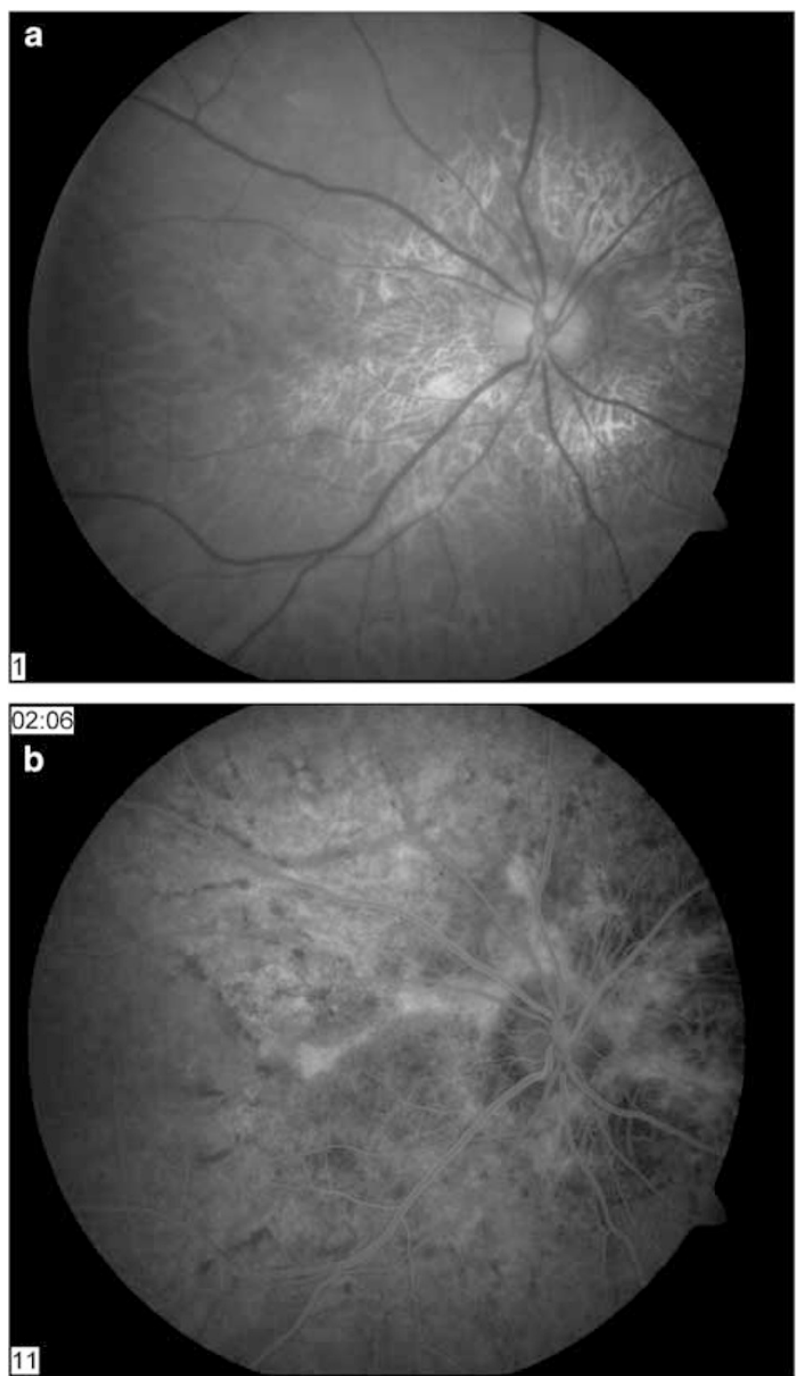

\section{OCT Image}

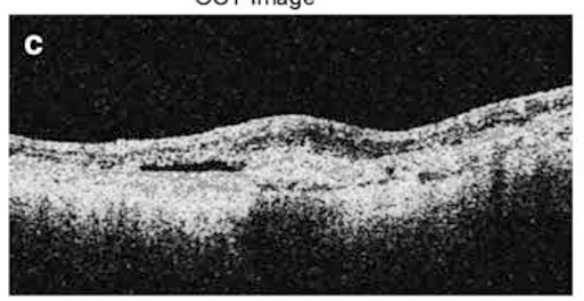

Fundus Image

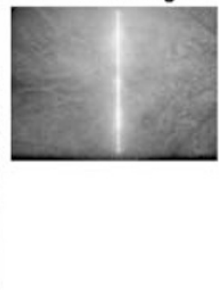

Figure 1 Imaging of the left eye: (a) Colour photograph shows angioid streaks and subfoveal CNV; (b) late FA shows mild late leakage underneath the fovea and angioid streaks; (c) OCT shows evidence of sub-retinal fluid.

agents in the treatment of $\mathrm{CNV}$, secondary to angioid streaks.

\section{References}

1 Spaide RF. Choroidal neovascularization in younger patients. Curr Opin Ophthalmol 1999; 10: 177-181.

2 Tewari A, Dhalla MS, Apte RS. Intravitreal bevacizumab for treatment of choroidal neovascularization in pathologic myopia. Retina 2006; 26: 1093-1094.

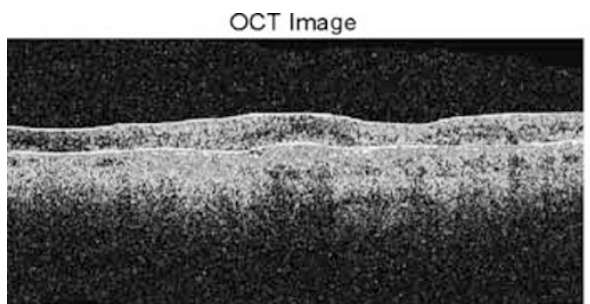

Fundus Image

Figure 2 OCT after three injections of bevacizumab shows resolution of sub-retinal fluid.

3 Freeman WR, Falkenstein I. Avastin and new treatments for AMD: where are we? Retina 2006; 26: 853-858.

4 Teixeira A, Moraes N, Farah ME, Bonomo PP. Choroidal neovascularization treated with intravitreal injection of bevacizumab (Avastin) in angioid streaks. Acta Ophthalmol Scand 2006; 84: 835-836.

RS Apte

Washington University School of Medicine,

Ophthalmology and Visual Sciences, Barnes Retina

Institute, St. Louis, MO, USA

E-mail: apte@vision.wustl.edu

Eye (2008) 22, 734-735; doi:10.1038/sj.eye.6702941; published online 14 March 2008

Sir

Bilateral congenital hamartomas of the retinal pigment epithelium in a patient with Down's syndrome Congenital hamartoma of the retinal pigment epithelium (RPE) was first described by Laqua ${ }^{1}$ in 1981, based on the clinical observation in two cases. Since then, not more than 20 unilateral cases of RPE hamartomas have been reported in the literature. We report a rare case of bilateral RPE hamartomas found in a patient with Down's syndrome.

\section{Case report}

A 48-year-old man with Down's syndrome came recently to the United Kingdom from abroad and was seen for the first time by an optician. He was found to have bilateral macular pigmented lesions in addition to significant refractive errors. Best-corrected visual acuity was difficult being around 6/24. Fundus examination revealed bilateral, jet black, and full-thickness retinal lesions located at the fovea (Figure 1). The diagnosis of bilateral RPE hamartomas was reported.

\section{Comment}

RPE hamartomas are focal, nodular, and jet-black lesions that usually appear to involve the full thickness of the retina and spill onto the inner retinal surface in an umbrella fashion. ${ }^{2}$ They occur at the macular area and are not associated with any changes in the surrounding tissues. Three patterns of involvement are described: superficial involvement of the retina, full-thickness retinal involvement with preretinal extension, or fullthickness retinal involvement with preretinal extension 


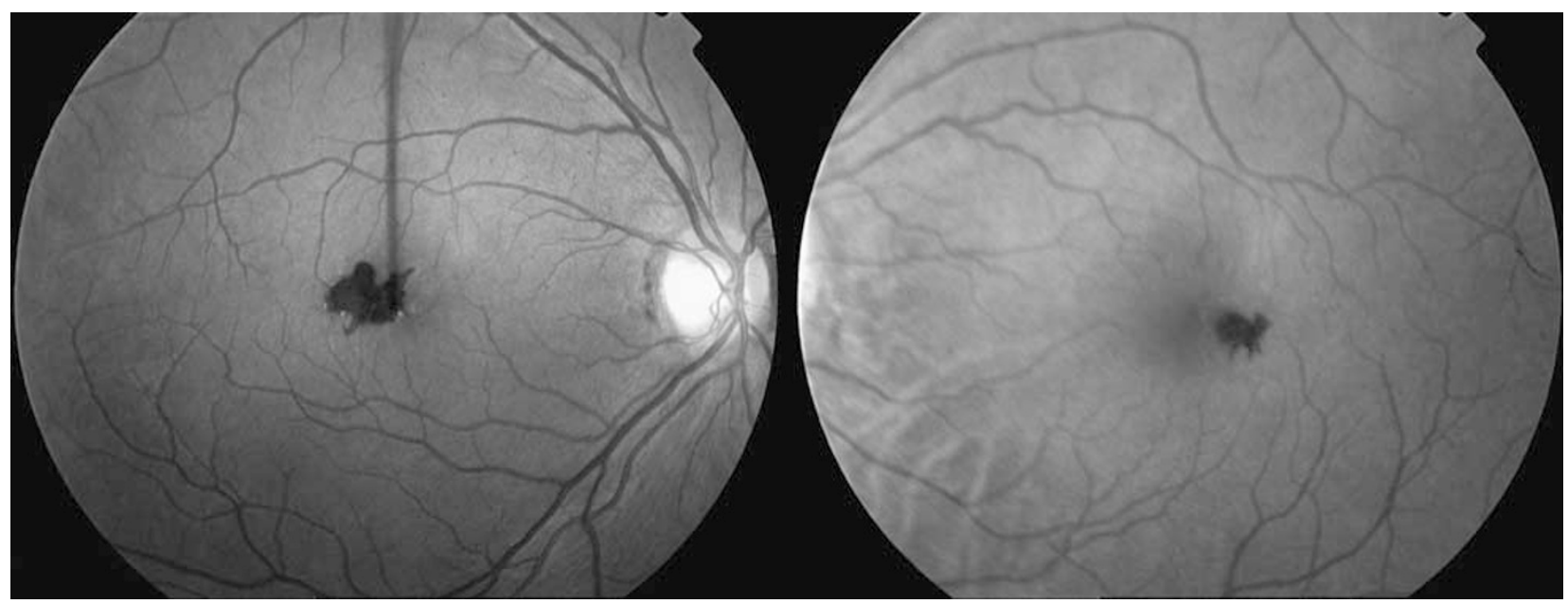

Figure 1 Bilateral RPE hamartoma on the macula.

and intrinsic vascularisation. Associated features commonly include minimally dilated retinal feeding vessels and surrounding retinal traction. ${ }^{3}$

Visual acuity is usually normal, but visual loss can occur from surrounding foveal traction or central foveal involvement. They generally remain stable without growth. The differential diagnosis includes congenital hypertrophy of the RPE, combined with hamartoma of the retina and RPE, adenoma or adenocarcinoma of the RPE, and RPE hyperplasia. Even though they are extremely infrequent, congenital RPE hamartomas have a very distinct appearance and can be recognised solely on their clinical features, most commonly being unilateral.

\section{References}

1 Laqua H. Tumors and tumor-like lesions of the retinal pigment epithelium. Ophthalmologica 1981; 183: 34-38.

2 Gass JD. Focal congenital anomalies of the retinal pigment epithelium. Eye 1989; 3: 1-18.

3 Shields CL, Shields JA, Marr BP, Sperber DE, Gass JD. Congenital simple hamartoma of the retinal pigment epithelium: a study of five cases. Ophthalmology 2003; 110: 1005-1011.

\section{A Panagopoulos, K Chalioulias and AT Murray}

Departmet of Ophthalmology, Birmingham and Midland Eye Centre, Birmingham, UK

E-mail: andypanagopoulos@yahoo.com

The authors have no proprietary interest in any of the products mentioned in this study

Eye (2008) 22, 735-736; doi:10.1038/eye.2008.27; published online 22 February 2008

Sir,

Hypotony and choroidal detachment as a complication of travoprost after trabeculectomy surgery

We present a case of recurrent choroidal detachment in a patient using travoprost, and suggest that prostaglandin analogues should be used with caution in patients who have undergone filtration surgery.

A 79-year-old man with primary open angle glaucoma underwent bilateral trabeculectomies in 1999. By 2003, his right bleb had failed, and latanoprost was prescribed to control his intraocular pressure (IOP). After 1 year, he returned with visual disturbance and right IOP of $4 \mathrm{mmHg}$, and was noted to have right 360-degree choroidal detachment (Figure 1). All topical treatment to the right eye was stopped; the choroidal detachment resolved over 5 months but the IOP rose to $25 \mathrm{mmHg}$, so travoprost was commenced. After 3 weeks, he again presented with right 360-degree choroidal detachment and IOP of $4 \mathrm{mmHg}$. Travoprost treatment was stopped, the choroidal detachment resorbed, but the IOP increased to $26 \mathrm{mmHg}$. A dorzolamide-timolol fixed combination was prescribed, maintaining IOP at $15 \mathrm{mmHg}$, with no sign of recurrent choroidal detachment to date.

Recommencement of topical ocular hypotensives following surgery is also known to precipitate hypotony and ciliochoroidal detachment. Discontinuation of topical therapy results in reattachment. ${ }^{1}$ Prostaglandin analogues increase uveoscleral outflow of aqueous, possibly by relaxation of the ciliary muscle associated with increased metalloproteinase activity. Choroidal detachment after the use of topical latanoprost has been reported previously. ${ }^{2,3}$ To our knowledge, choroidal detachment has not been previously reported with topical travoprost after glaucoma filtration surgery, although there are reports of choroidal detachment in eyes with chronic angle closure, but no history of glaucoma surgery. ${ }^{4}$ As the uveoscleral pathway is pressure-independent, it has been suggested that prostaglandin analogues could lower IOP below episcleral venous pressure, and thereby cause hypotony and choroidal detachment. ${ }^{5}$ We, therefore, suggest that in patients who have undergone previous glaucoma surgery who subsequently need topical antihypotensive medication, prostaglandin analogues should be used with caution, as they may precipitate hypotony and choroidal detachment. 